\title{
Nasal packing aspiration in a patient with Alzheimer's disease: a rare complication
}

This article was published in the following Dove Press journal:

International Journal of General Medicine

26 July 2012

Number of times this article has been viewed

Eleftherios Koudounarakis

Nikolaos Chatzakis

loannis Papadakis

Irene Panagiotaki

George Velegrakis

Department of Otorhinolaryngology, University Hospital of Heraklion, Crete, Greece
Correspondence: Eleftherios

Koudounarakis

Ethnikis Antistaseos 58, Heraklion,

Crete 71306, Greece

Tel +306973232477

Email ekoudounarakis@hotmail.com
Abstract: Nasal bleeding is a frequent problem for patients receiving anticoagulant agents. Most cases are successfully managed with anterior or posterior nasal packing. However, the complications of nasal packing should be always considered. We report the case of a 78-year-old man with Alzheimer's disease who was treated for anterior epistaxis with anterior nasal packing using three pieces of antibiotic-soaked gauze. Two days later, the patient was admitted to the emergency department in respiratory distress. A chest x-ray demonstrated atelectasis of the right lung. During an examination of the nasal cavities, the nasal packing was removed, and one of the gauze pieces was missing. The patient underwent rigid bronchoscopy, and the missing gauze was found to be obstructing the right main bronchus. The patient's respiratory function improved considerably after removal of the foreign body. It is assumed that gauze packs should be used with caution in patients with an impaired level of consciousness and neurodegenerative diseases.

Keywords: epistaxis, nasal packing, aspiration, Alzheimer's disease, cough reflex

\section{Introduction}

Epistaxis is a common occurrence, especially in patients receiving anticoagulant agents. Approximately $60 \%$ of the adult population will experience at least one episode of epistaxis throughout their lifetime. ${ }^{1}$ However, only $5 \%-10 \%$ will require medical assistance. It is estimated that approximately $90 \%-95 \%$ of all epistaxis occurs in the anterior part of the nasal septum, in Kiesselbach's plexus. Anterior nasal bleeding is usually a self-limiting condition or can be safely managed with chemical or electrical cautery and anterior nasal packing, even in the primary care setting. ${ }^{2}$ Posterior nasal bleeding is a more difficult issue, and many methods and surgical techniques have been developed for its management. When nasal packing is used, the physician should always consider its complications, such as acute sinusitis, toxic shock syndrome, nasal septum necrosis, and perforation, as well as some rare complications that have been reported in the literature, including bowel perforation following ingestion of a nasal pack, alar region necrosis, and the formation of a pyogenic granuloma. ${ }^{3-6}$ In cases of failure of posterior nasal packing, cauterization of the sphenopalatine artery under endoscopic control is frequently used with good results. ${ }^{7}$ Additionally, the Nd-Yag laser is a valuable tool for the treatment of epistaxis in patients with hereditary hemorrhagic telangiectasia. ${ }^{8}$ The choice of treatment depends on the available equipment and the skills of the clinician. In this paper, a rare case of foreign body aspiration is presented as a complication of anterior nasal packing in a patient with Alzheimer's disease. 


\section{Case report}

A 78-year-old man presented to a primary care unit with epistaxis from the left nasal cavity. The patient's medical history included hypertension, hyperlipidemia, and Alzheimer's disease. He was receiving an antiplatelet agent (acetylsalicylic acid) as prophylaxis for coronary artery disease. A clinical examination revealed anterior nasal bleeding that was managed successfully with anterior nasal packing using three $2 \times 10 \mathrm{~cm}$ antibiotic-soaked gauze packs in the left nasal cavity. Two days later, the patient was admitted to the emergency department of our hospital in respiratory distress with tachypnea and tachycardia. His initial vital signs included a blood pressure of 173/98 mmHg, a pulse of 117 beats per minute, a respiratory rate of 26 breaths per minute, and oxygen saturation of $86 \%$ on room air. Examination of the respiratory system revealed absent breath sounds over the right hemithorax, with a dull sound during percussion. The chest X-ray demonstrated atelectasis of the right lung and retraction of the mediastinum to the right. Due to the recent history of nasal bleeding and anterior nasal packing, both nasal cavities were inspected. The nasal packing was removed, and a gauze pack was missing. Although no episode of choking or intense cough was witnessed by the patient's family during the last 2 days, a suspicion for foreign body aspiration arose because of the missing gauze pack. For this reason, the patient underwent rigid bronchoscopy under general anesthesia, and during the procedure, a foreign body was found to obstruct the right main bronchus. The foreign body, which consisted of the missing gauze pack saturated with mucus and blood clots, was removed successfully.

Due to his critical state, the patient was transferred intubated to the intensive care unit, where his respiratory function improved considerably, and intravenous antibiotics were also administered. On the second day of hospitalization in the intensive care unit, epistaxis from the left nasal cavity recurred, and anterior rhinoscopy revealed anterior nasal bleeding, which was successfully controlled using electrocautery without the need for nasal packing. The patient recovered completely and was discharged after 12 days of hospitalization.

\section{Discussion}

Epistaxis is one of the most common emergency conditions, representing approximately $9.5 \%-16 \%$ of all otorhinolaryngologic emergencies, with a male predominance. ${ }^{9}$ Initial management of the unstable patient with bleeding begins by assessing airway, breathing, and circulation (the $\mathrm{ABCs}$ ). Identification of the bleeding point is the next important step.
The source of most anterior epistaxis episodes can be identified using a headlight and adequate suction. Once the bleeding point is identified, cauterization, either chemical (silver nitrate) or electrical, usually provides definitive treatment. ${ }^{2}$ If cauterization is unsuccessful, anterior nasal packing is used. Nasal packing acts by direct pressure to the nasal septum, reduction of mucosal irritation and clot formation that enhances pressure. A variety of materials are used for nasal packing, most commonly antibiotic-soaked gauze packs and nasal tampons, with or without airways. ${ }^{10}$ The urgent nature of epistaxis requires the physician to have a thorough knowledge of the anatomy of the nasal cavity, the modalities available to control the epistaxis and the potential complications of those modalities. In addition, training is required for effective nasal packing, because this procedure can be difficult and traumatic for the patient.

Various complications are related to nasal packing. ${ }^{3}$ The most common complication is that removal of the packing dislodges healing tissue and causes recurrence of bleeding. Nasal packing can also cause a lack of oxygen in patients who have difficulty breathing through their mouths, such as those with chronic obstructive pulmonary disease, leading to a drop in blood oxygen content and an increase in blood carbon dioxide levels. This, in turn, can cause respiratory and cardiac complications, such as a racing pulse. Patients with respiratory compromise may require airways control and mechanical ventilation, whereas patients with hemodynamic compromise require volume and blood product resuscitation.

In the case of this patient, one of the gauze packs used for nasal packing migrated from the nasal cavity to the lower airway, although no event of choking or intense cough was witnessed by the patient's family. A case of gauze ribbon migration with partial obstruction of the glottis has been reported in a patient with facial trauma and an impaired level of consciousness. However, the migrated nasal packing was recognized and removed shortly thereafter without any further complications. ${ }^{11}$

Impairment of the cough reflex may have played an important role in the clinical presentation of this patient. The cough reflex is a primary airway defense mechanism and a common symptom in patients with foreign body aspiration. It has been reported that elderly patients with aspiration pneumonia have a downregulation of the cough reflex and depression of the urge to cough, which in turn are attributed to supramedullary dysfunction. ${ }^{12}$ Patients with Alzheimer's disease have also been shown to have high rates of silent aspiration, as demonstrated with video fluoroscopy. ${ }^{13}$ 
In this group of patients, if anterior nasal packing is required, the use of nasal tampons tethered to the outside of the nose by suture seems to be safer. However, a case of losing even that type of nasal pack after closed reduction of a nasal bone fracture has been reported in a patient who was mentally impaired and did not know whether he had swallowed the pack. ${ }^{14}$ Therefore, quilting an X-ray indicator from surgical gauze into the nasal tampon has been proposed to detect the missing nasal pack.

Before using a nasal pack, the clinician should attempt chemical or electrical cautery once the bleeding point is identified because this usually provides definitive treatment without the need for nasal packing. ${ }^{2}$ However, the increasing use of oral anticoagulants results in high rates of intractable epistaxis, requiring more aggressive local hemostasis measures and longer inpatient stays. ${ }^{15}$

In conclusion, patients with an impaired level of consciousness and neurodegenerative diseases have an increased risk of aspiration. Gauze packs should be used with caution for the treatment of nasal bleeding in this group of patients because the migration and aspiration of such a gauze pack can be a potentially fatal complication.

\section{Disclosure}

The authors report no conflict of interest in this work.

\section{References}

1. Monux A, Tomás M, Kaiser C, Gavilán J. Conservative management of epistaxis. J Laryngol Otol. 1990;104(11):868-870.

2. Douglas R, Wormald PJ. Update on epistaxis. Curr Opin Otolaryngol Head Neck Surg. 2007;15(3):180-183.
3. Schaitkin B, Strauss M, Houck JR. Epistaxis: medical versus surgical therapy: a comparison of efficacy, complications, and economic considerations. Laryngoscope. 1987;97(12):1392-1396.

4. Hashmi SM, Gopaul SR, Prinsley PR, Sansom JR. Swallowed nasal pack: a rare but serious complication of the management of epistaxis. J Laryngol Otol. 2004;118(5):372-373.

5. Civelek B, Kargi AE, Sensöz O, Erdogan B. Rare complication of nasal packing: alar region necrosis. Otolaryngol Head Neck Surg. 2000; 123(5):656-657.

6. Sheen TS, Ko JY, Hsu YH. Pyogenic granuloma - an uncommon complication of nasal packing. Am J Rhinol. 1997;11(3):225-227.

7. Wiorowski M, Schultz P, Perrot JB, Gentine A, Debry C. Indications and results of cauterization by endoscopic approach of the sphenopalatine artery in severe posterior epistaxis. Auris Nasus Larynx. 2004;31(2): 131-133.

8. Velegrakis GA, Prokopakis EP, Papadakis CE, Helidonis ES Nd:YAG laser treatment of recurrent epistaxis in heredity hemorrhagic telangiectasia. J Otolaryngol. 1997;26(6):384-386.

9. Monjas-Cánovas I, Hernández-García I, Mauri-Barberá J, Sanz-Romero B, Gras-Albert JR. Epidemiology of epistaxis admitted to a tertiary hospital. Acta Otorrinolaringol Esp. 2010;61(1):41-47. Spanish.

10. Gupta M, Singh S, Chauhan B. Comparative study of complete nasal packing with and without airways. B-ENT. 2011;7(2):91-96.

11. Helwani M, Saied NN, Foroughi V. Management of airway obstruction caused by nasal packing material in a trauma patient. $J$ Clin Anesth. 2006;18(1):50-51.

12. Yamanda $\mathrm{S}$, Ebihara $\mathrm{S}$, Ebihara $\mathrm{T}$, et al. Impaired urge-to-cough in elderly patients with aspiration pneumonia. Cough. 2008;4:11.

13. Garon BR, Sierzant T, Ormiston C. Silent aspiration: results of 2,000 video fluoroscopic evaluations. J Neurosci Nurs. 2009;41(4):178-185.

14. Kim MG, Baek RM, Minn KW, Heo CY, Kwon SS, Park CY. Nasal packs with X-ray indicators. Ann Plast Surg. 2006;56(3):342-343.

15. Smith J, Siddiq S, Dyer C, Rainsbury J, Kim D. Epistaxis in patients taking oral anticoagulant and antiplatelet medication: prospective cohort study. J Laryngol Otol. 2011;125(1):38-42.
International Journal of General Medicine

\section{Publish your work in this journal}

The International Journal of General Medicine is an international peer-reviewed open-access journal that focuses on general and internal medicine, pathogenesis, epidemiology, diagnosis, monitoring and treatment protocols. The journal is characterized by the rapid reporting of reviews, original research and clinical studies across all disease areas.

\section{Dovepress}

A key focus is the elucidation of disease processes and management protocols resulting in improved outcomes for the patient. The manuscript management system is completely online and includes a very quick and fair peer-review system. Visit http://www.dovepress.com/ testimonials.php to read real quotes from published authors. 